\title{
Isolation, characterization and expression of $11 \beta$-hydroxysteroid dehydrogenase type 2 cDNAs from the testes of Japanese eel (Anguilla japonica) and Nile tilapia (Oreochromis niloticus)
}

\author{
J Q Jiang ${ }^{1}$, D S Wang ${ }^{1}$, B Senthilkumaran ${ }^{1,2}$, T Kobayashi ${ }^{1,2}$, H K Kobayashi ${ }^{1,2}$, \\ A Yamaguchi ${ }^{1,2}, \mathrm{~W} \mathrm{Ge}^{3}$, G Young ${ }^{4}$ and $\mathrm{Y}$ Nagahama $^{1,2}$ \\ ${ }^{1}$ Laboratory of Reproductive Biology, National Institute for Basic Biology, Okazaki 444-8585, Japan \\ ${ }^{2}$ CREST, Kawaguchi, Saitama 332-0012, Japan \\ ${ }^{3}$ Department of Biology, The Chinese University of Hong Kong, Shatin, N.T., Hong Kong, China \\ ${ }^{4}$ Department of Biological Sciences and Center for Reproductive Biology, University of Idaho, Moscow, Idaho 83844, USA \\ (Requests for offprints should be addressed to Y Nagahama, Laboratory of Reproductive Biology, Department of Developmental Biology, \\ National Institute for Basic Biology, Okazaki 444-8585, Japan; Email: nagahama@ nibb.ac.jp) \\ (J Q Jiang is now at Department of Zoology, University of Oxford, Oxford OX1 3PS, UK) \\ (J Q Jiang and D S Wang contributed equally to this work)
}

\begin{abstract}
The Japanese eel (Anguilla japonica) and Nile tilapia (Oreochromis niloticus) 11ß-hydroxysteroid dehydrogenase type $2(11 \beta-H S D 2)$ cDNAs were isolated from their respective testes cDNA libraries. The cDNAs predict two peptides of 436 and 406 amino acid residues that share about $42 \%$ homology with mammalian $11 \beta-H S D$ type 2 proteins. Analysis of the tissue distribution pattern by RT-PCR reveals that $11 \beta$-HSD2 is expressed in a wide variety of tissues in tilapia, with higher expression in kidney and gill of

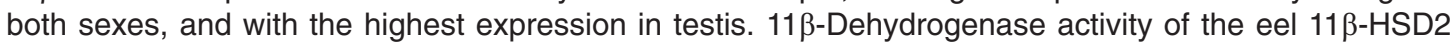
was confirmed by demonstrating the conversion of cortisol to cortisone by the recombinant protein after transient expression of this cDNA clone in COS-1 cells. Bands of $\sim 2.7$ and $\sim 3.8 \mathrm{~Kb}$ were detected in Northern blot of eel and tilapia testes respectively, which is consistent with the cloned cDNA sizes of the two species. Northern blot analysis also revealed that the expression of the eel testis $11 \beta-H S D 2$ gene could be induced by human chorionic gonadotropin (hCG) injection, implying a role of $11 \beta-\mathrm{HSD} 2$ in hCG-induced 11-ketotestosterone production and spermatogenesis in the Japanese eel.
\end{abstract}

Journal of Molecular Endocrinology (2003) 31, 305-315

\section{Introduction}

11-Ketotestosterone (11-KT) is a major androgen in fish. Using a testis organ culture system, we showed that hormonal induction of spermatogenesis in the testis of the Japanese eel (Anguilla japonica) involves gonadotropin stimulation of Leydig cells to produce 11-KT which, in turn, activates Sertoli cells to stimulate premitotic spermatogonia to complete spermatogenesis (Miura et al. 1991a,b,c, Nagahama 1994). 11 -Ketotestosterone is synthesized from testosterone by the actions of two enzymes, $11 \beta$-hydroxylase $(\mathrm{P} 450(11 \beta))$ and $11 \beta$-hydroxysteroid dehydrogenase

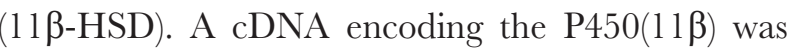
isolated from the Japanese eel testis and its expression was investigated in testes during human chorionic gonadotropin (hCG)-induced spermatogenesis (Jiang et al. 1996). Recently, P450(11ß) cDNAs were also isolated from rainbow trout (Liu et al. 2000, Kusakabe et al. 2002) and the Nile tilapia (D S Wang, B Senthilkumaran, T Kobayashi and Y Nagahama, unpublished data). It is important to further investigate the role of $11 \beta$-HSD (the final enzyme in the biosynthetic pathway of $11-\mathrm{KT}$ ) in hCG-induced 11-KT production and spermatogenesis in the Japanese eel and other fish species. 
Until recently, cloning of $11 \beta-\mathrm{HSD}$ cDNA has been reported only for several mammals. Two different $11 \beta$-HSD enzymes have been described. The type $111 \beta$-HSD (11 $\beta$-HSD 1) was first isolated and cloned from liver microsomes of rat (Agarwal et al. 1989, Monder \& Lakshmi 1989). This type of enzyme is an NADP-dependent enzyme with a relatively low affinity for cortisol or corticosterone. The type $111 \beta$-HSD was also cloned from human (Tannin et al. 1991), mouse (Rajan et al. 1995), pig (AF414124), cow (AF548027), squirrel monkey (Moore et al. 1993) and sheep (Yang et al. 1992) tissues. The type $211 \beta$-HSD (11 $\beta$-HSD2) has been cloned from human (Albiston et al. 1994), sheep (Agarwal et al. 1994), rat (Zhou et al. 1995), mouse (Cole 1995), pig (AF374414), cow (Romero et al. 2000) and horse (AF126744) kidney and rabbit collecting duct cells (Naray-Fejes-Toth \& Fejes-Toth 1995). This type of $11 \beta$-HSD is NAD-dependent and has a higher affinity in the nanomolar range for corticosterone and cortisol, and co-localizes with the mineralocorticoid receptor (Naray-Fejes-Toth et al. 1991, Rusvai \& Naray-Fejes-Toth 1993, Krozowski et al. 1994, Tomlinson \& Stewart 2001). These two types of $11 \beta$-HSD belong to the short-chain dehydrogenase/ reductase (SDR) superfamily. The homology in amino acid sequence is only about $18 \%$, indicating that they are products of different genes (NarayFejes-Toth \& Fejes-Toth 1995). More recently, the cloning and characterization of rainbow trout $11 \beta$ HSD2 was reported (Kusakabe et al. 2003).

In this study, our aim is to investigate gonadotropin regulation of $11 \beta$-HSD gene expression as well as to compare evolutionary and structural aspects of this enzyme. We report here the isolation of two full-length cDNAs encoding 11 $\beta$-HSD2 from eel and tilapia testes cDNA libraries. The enzyme activity of eel $11 \beta$-HSD expressed in COS-1 cells was analyzed. In addition, eel $11 \beta$-HSD gene expression in the testis during hCG-induced spermatogenesis and the distribution of $11 \beta$-HSD mRNA in different tissues were studied.

\section{Materials and methods}

\section{Isolation of eel and tilapia 11ß-HSD cDNAs}

Based on 11ß-HSD sequences of mammals, a conserved five amino acid sequence, MEVNF, was selected and its mixed degenerate oligonucleotides, 5'-ATGGA(AG)GT(ACGT)AA(CT) TT(CT)-3', were synthesized. The synthesized oligonucleotides were used as a probe by labeling the $5^{\prime}$ ends with $\gamma-{ }^{32} \mathrm{P}$-ATP using T4 polynucleotide kinase (Takara, Otsu, Shiga, Japan). A cDNA library from testes of Japanese eels sampled 3 days after hCG injection was constructed in $\lambda$ ZAP II. A total of 200000 phages from this library were screened with low stringency. One positive clone was isolated and excised. Sequence analysis of the cDNA insert indicated that it was similar to mammalian $11 \beta$-HSD type 2 , but truncated at the $5^{\prime}$ end. This cDNA insert was then labeled by ${ }^{32} \mathrm{P}-\mathrm{dCTP}$ using the Random Extension Plus kit (Dupont, Wilmington, DE, USA) to screen the cDNA library again. Another 200000 phages were screened. The hybridization and the subsequent wash were conducted this time at high stringency. Ten positive clones were isolated, excised and sequenced. Nest deletion was performed to delete the cDNA insert from both ends using the ExoIII/Mung bean nuclease deletion system.

In order to obtain tilapia $11 \beta$-HSD2, based on eel and mammalian 11 -HSD2 sequences a pair of degenerate primers, forward primer $(F w), 5^{\prime}$-GG (TG)CTGTGGGG(ACT)CT(GT)GT(GCT)AA GAA and reverse primer $(R v)$, 5'-GCTGG(TC) TT(GG)GAGG(TG)(TG)CGATA, were designed for RT-PCR. A 236 bp tilapia 11 $\beta$-HSD2 fragment was obtained, sub-cloned and sequenced. This fragment was labeled by ${ }^{32} \mathrm{P}-\mathrm{dCTP}$ and used as a probe to screen the tilapia testis library using the procedures described above. Fifteen positive clones were isolated, excised and sequenced. All cDNA sequence analyses were carried out using either an ABI 373 or an ABI 377 DNA Sequencer (Applied Biosystems, Foster City, CA, USA). The sequences were submitted to GenBank under the accession nos AB097668 (eel 11ß-HSD2) and AY190043 (tilapia 11ß-HSD2).

\section{Phylogenetic analysis}

Homology analyses of $11 \beta$-HSD2 protein were performed with Lasergene software. The multiple alignment software ClustalX (Thompson et al. 1997) was employed to calculate and depict trees by the N-J method using human $11 \beta-H S D 1$ as an outgroup. The fugu $11 \beta$-HSD2 protein sequences used in the alignment were obtained from the fugu 
(Takifugu rubripes) genome (http://www.ncbi.nlm. nih.gov/PMGifs/Genomes/fugu.html). Initially, fugu $11 \beta$-HSD2 genomic sequences were obtained by

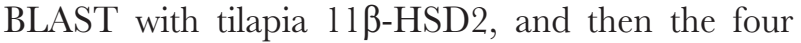
intron sequences were deleted to deduce the protein sequence.

\section{Analysis of 11ß-HSD2 mRNA tissue distribution by RT-PCR}

Total RNA $(5 \cdot 0 \mu \mathrm{g})$ was isolated from various tissues of adult tilapia containing either postvitellogenic ovary or spermiating (sperm expressed when gentle pressure was applied to the abdomen) testis and treated with DNase I (Invitrogen, Carlsbad, CA, USA). The cDNAs were then synthesized and RT-PCR was employed for the analysis of tilapia $11 \beta$-HSD2 expression. The PGR reaction consisted of $2 \mathrm{~min}$ at $94{ }^{\circ} \mathrm{C}$, followed by 33 cycles of $94{ }^{\circ} \mathrm{C}(30 \mathrm{~s}), 55^{\circ} \mathrm{C}(30 \mathrm{~s})$, and $72{ }^{\circ} \mathrm{C}$ (1 min), ending with $10 \mathrm{~min}$ extension at $72^{\circ} \mathrm{C}$. The following two gene specific primers, which are located in exons 3 and 5 respectively were used to amplify $541 \mathrm{bp}$ cDNA fragments by PCR: $(F w)$ 5'-AATAACGCGGGCGTGTGGGTGAAC$3^{\prime}$ and $(R v)$ 5'-GGCTCAAGCGAGAGCCTGCA AAGT-3'.

A 342 bp tilapia $\beta$-actin fragment was amplified to test the quality of the cDNA used in the PGR reaction with a pair of $\beta$-actin primers specific for the Nile tilapia, $(F w)$ 5'-GGCATCACACCTTCT ACAACGA-3' and $(R v)$ 5'-ACGGTCTGTCAGG ATCTTCA-3'. All the PCR products were electrophoresed using $1.5 \%$ agarose gels and the gels were stained with ethidium bromide to visualize bands.

\section{Transient expression and enzymatic activity}

The eel $11 \beta$-HSD2 cDNA insert was cut out of the multicloning sites of pBluescript by SpeI and HindIII and inserted into the same sites in the expression vector pBK-CMV. The constructed recombinant expression vector was named pBKE11 HSD. The construct was transfected into COS-1 cells using LipofectAMINE reagent (GibcoBRL, Gaithersburg, MD, USA) according to the supplier's instructions. Seventy-two hours after transfection, the COS-1 cells were incubated with $16 \mathrm{pmol}{ }^{3} \mathrm{H}$-labeled cortisol $(2 \cdot 33 \mathrm{TBq} / \mathrm{mmol}$, Amersham, Buckinghamshire, UK) in triplicate.
After $2 \mathrm{~h}$ incubation, the medium was collected, extracted twice with equal volumes of diethyl ether and the extracts were dried. The samples were dissolved in acetone, mixed with authentic cortisol and cortisone, spotted onto a TLC plate (64 F254, Merck, Lindenplatz, Haar, Germany) and developed in chloroform and acetone (82:18, vol/vol). The cold steroids were detected under UV light and served as mobility markers. The plate was then exposed to ${ }^{3} \mathrm{H}-\mathrm{Hyp} e r f i l m$ (Amersham). For calculating the percentage conversion of cortisol to cortisone, both cortisol and cortisone bands were scraped from the thin-layer chromatography plate, eluted from the silica with $0.5 \mathrm{ml}$ isopropanol, and separated from the silica by centrifugation. The isopropanol solution was decanted into vials for scintillation counting.

\section{RNA isolation and Northern blotting}

Eels were injected with hCG (Teikoku Zoki, Tokyo, Japan) at a dosage of $5 \mathrm{IU} / \mathrm{g}$. Total RNA was isolated from testes of eels killed from 1 to 18 days post hCG injection or from untreated eels. Ten micrograms total RNA from each sample were separated on a $1 \%$ formaldehyde-agarose denaturing gel, transferred to Hybond- ${ }^{+}$nylon membrane (Amersham) and baked at $80{ }^{\circ} \mathrm{C}$ for $2 \mathrm{~h}$. The full cDNA insert of eel $11 \beta$-HSD2 was labeled with ${ }^{32}$ P-dCTP using the Random Extension Plus kit (Dupont). Hybridization was performed at $60{ }^{\circ} \mathrm{C}$ in a hybridization solution containing $6 \times \mathrm{SSC}$, $5 \times$ Denhardt's solution, $1 \%$ SDS and $200 \mu \mathrm{g} / \mathrm{ml}$ denatured herring sperm DNA. After $20 \mathrm{~h}$ hybridization, the membrane was washed three times at $60{ }^{\circ} \mathrm{C}$ with a series of SSC-SDS buffer. The membrane was exposed to an imaging plate, and the hybridization signals were analyzed by a BAS 2000 Bio-Image Analyzer. To perform Northern blot analysis in tilapia, total RNA was extracted from post-vitellogenic ovary and spermiating testis using ISOGEN (Nippon Gene, Toyohama, Japan). Poly(A) ${ }^{+}$RNAs were purified using Oligotex-DT30 (Takara), and subsequently separated by electrophoresis and hybridized with a fragment of tilapia $11 \beta$-HSD2 cDNA following the method described above. The membrane was stripped and further hybridized with ${ }^{32} \mathrm{P}$-labeled tilapia $\beta$-actin probe. Subsequently, densitometry measurements were carried out for each band detected. The eel and tilapia 11ß-HSD2 relative 
mRNA levels were calculated as the ratio to $\beta$-actin and expressed as the percentage of respective controls.

\section{Results}

\section{Nucleotide and amino acid sequence of eel and tilapia 11ß-HSD2}

The cloned $11 \beta$-HSD cDNAs from eel and tilapia are 2689 and 3804 bp respectively. The full length eel cDNA consists of a $69 \mathrm{bp} 5^{\prime}$-untranslated region (UTR), a 1308 bp open reading frame (ORF) which predicts a protein of 436 amino acids, and a $1312 \mathrm{bp} \mathrm{3'-UTR} \mathrm{with} \mathrm{a} 23 \mathrm{bp}$ poly(A) tail. The tilapia cDNA consists of a 223 bp 5'-UTR, a $1221 \mathrm{bp}$ ORF which predicts a protein of 406 amino acids, and a $2361 \mathrm{bp} \mathrm{3'-UTR} \mathrm{with} \mathrm{a} 18 \mathrm{bp}$ poly(A) tail. Both cDNAs possess polyadenylation signals.

\section{Phylogenetic analysis}

A BLAST search against the GenBank nucleotide and protein databases indicated that the amino acid sequences encoded by these cDNAs are similar to mammalian $11 \beta$-HSD type 2 , belonging to the SDR superfamily. Alignments of their amino acid sequences depicted in Fig. 1 revealed two motifs in both eel and tilapia $11 \beta-H S D 2$ which are characteristic of $11 \beta$-HSD2. One (box I) near the amino terminus most likely represents the NAD-binding site. The other (box II) contains tyrosine and lysine residues. These two amino acids are highly conserved in all short-chain alcohol dehydrogenase enzymes. The conserved region in box II is probably important for catalytic activity. Alignments of these amino acid sequences also revealed a region consisting of 47 amino acids in eel $11 \beta$-HSD2 that had no counterparts in the sequences of tilapia, rainbow trout, fugu and mammals $11 \beta$-HSD2s. Eel $11 \beta$-HSD2 is also 19 amino acids shorter than the other fish and mammalian 11 $\beta$-HSD2s in the N-terminus (Fig. 1). Amino acid sequence similarity among $11 \beta$-HSD2 of eel, tilapia, other fish species and mammals are shown in Table 1. Generally, fish $11 \beta$-HSD2s show 60-70\% similarity among themselves and show about $40-45 \%$ similarity to those of mammals. However, the cloned eel and tilapia $11 \beta$-HSDs only show around $20 \%$ similarity to the human and other mammalian $11 \beta$-HSD 1 s.
Based on the alignments of the amino acid sequences, a phylogenetic tree of all the cloned $11 \beta$-HSD2s from fish and mammals was constructed with human $11 \beta$-HSDl as an outgroup (Fig. 2). The cloned eel and tilapia 11ß-HSDs showed much longer phylogenetic distance to the

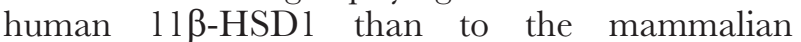
$11 \beta$-HSD2s. Fish 11 $\beta$-HSD2s formed a group which is also relatively far from the mammalian group in phylogenetic distance. Among the four fish species, tilapia and fugu $11 \beta$-HSD 2 s constitute one clade different from that formed by the eel and trout proteins.

\section{Tissue distribution of eel and tilapia 11 $\beta$-HSD mRNA}

Analysis of the tissue distribution pattern of $11 \beta$-HSD2 in tilapia revealed that the gene is expressed in a wide variety of tissues with higher expression in kidney, gill and intestine. The highest expression level was found in testis (Fig. 3). Further, positive and negative controls validated the distribution pattern. A similar pattern of expression was also found in Japanese eel by RT-PCR (data not shown).

\section{Enzymatic activity in COS- 1 cells}

COS-1 cells transfected with pBK-GMV/E11 HSD converted large amounts of cortisol into cortisone after only $2 \mathrm{~h}$ incubation with ${ }^{3} \mathrm{H}$-labeled cortisol. A clear ${ }^{3} \mathrm{H}$-labeled band corresponding to cortisone was visualized by TLG of extracts of media from pBK-GMV/E11 HSD transfected COS-1 cells but not from extracts of media from COS-1 cells transfected with the pKB-CMV vector. After $2 \mathrm{~h}$ incubation, COS-1 cells transfected with pBK-GMV/E11 HSD had converted $50 \%$ of ${ }^{3} \mathrm{H}$-labeled cortisol to cortisone (Fig. 4).

\section{1ß-HSD2 mRNA levels during hCG-induced spermatogenesis in eel and in natural maturation in Nile tilapia}

Northern blot analysis revealed a single transcript of about $2.7 \mathrm{~kb}$ present in eel testis. The hybridization signal could not be detected from eel testis on day 0 (before hCG injection), but appeared at day 1 , and peaked in density at day 3 post hCG injection. The hybridization signals 


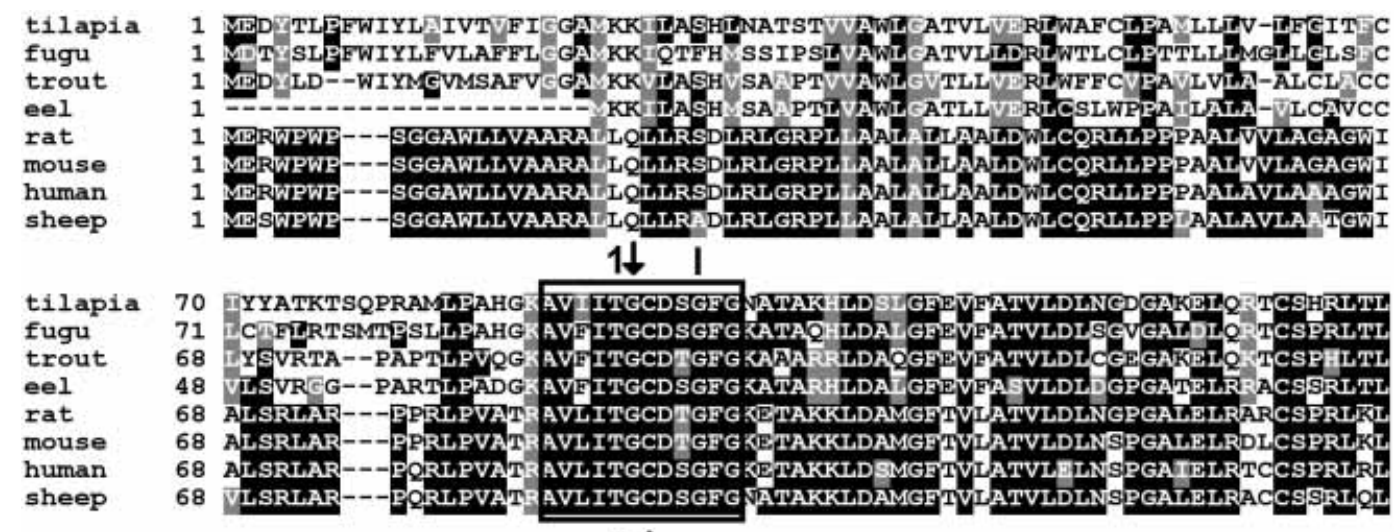

\section{$2 \downarrow$}

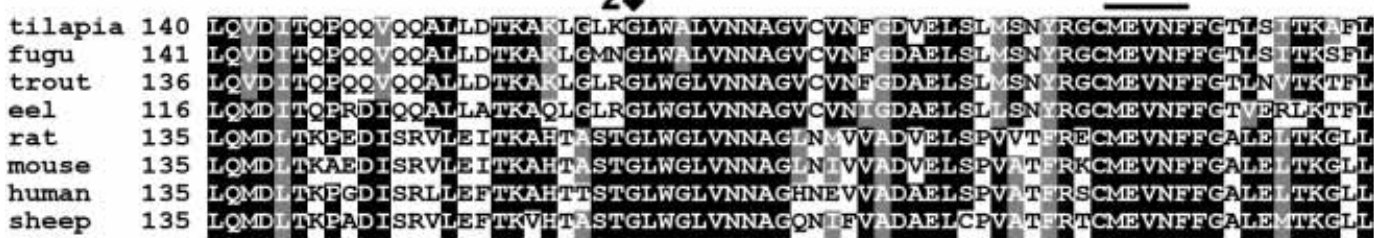

\section{$3 \downarrow \quad$ II}

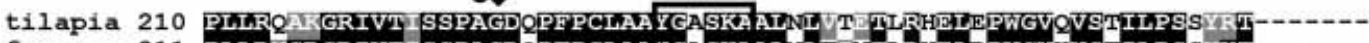

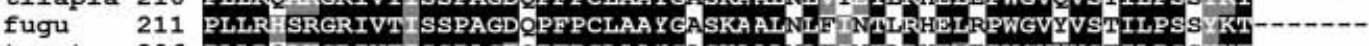

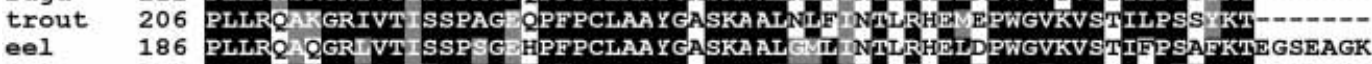

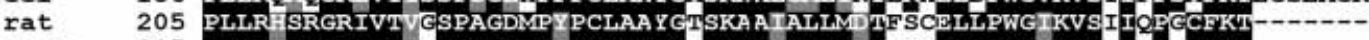
mouse 205 PLLR: SRGRIVT GSPAGDMP YCLAA Y IISKA A ALLMDTFGCELLPWG IKVSI IKE GCFKT--.---

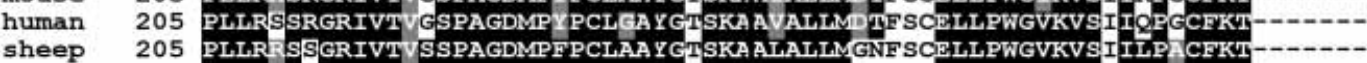

sheep 205 PLLR:SSGRIVTVSSPAGDMPFPCLAA YGTSKA ALALIMGN
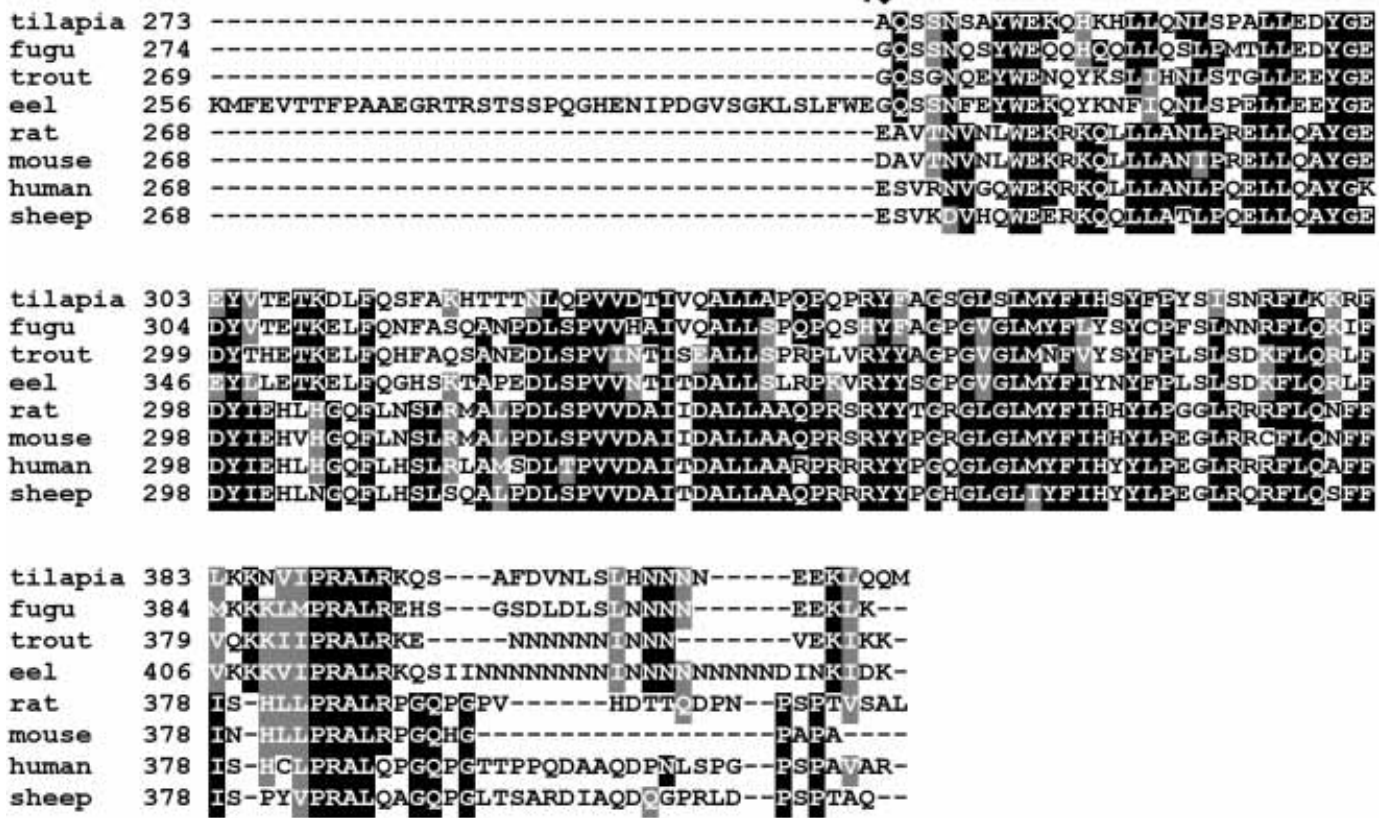

Figure 1 Alignment of the amino acid sequences of fish and mammalian $11 \beta-H S D 2 s$. BOXSHADE

(http;//www.ch.embnet.org/ software/BOX_form.html) was used to make this figure. The conserved five amino acid sequence, MEVNF, is marked by a solid line above. The four intron positions are numbered and marked by arrows. Boxes I and II are the putative NAD-binding domain and the catalytic site respectively. For GenBank accession numbers refer to the legend of Fig. 2. 
Table 1 Amino acid similarities (shown as \%) between eel and tilapia 11/-HSD2 and those of rainbow trout, fugu and mammals

\begin{tabular}{|c|c|c|c|c|c|c|c|c|c|}
\hline & Eel & Tilapia & Trout & Fugu & Sheep & Mouse & Rat & Human & Human-1* \\
\hline Eel & $\overline{100 \cdot 0}$ & $59 \cdot 2$ & $70 \cdot 3$ & $58 \cdot 6$ & $42 \cdot 8$ & 43.5 & $43 \cdot 2$ & $42 \cdot 7$ & $19 \cdot 9$ \\
\hline Tilapia & 59.2 & $100 \cdot 0$ & $66 \cdot 2$ & 70.9 & 41.3 & $42 \cdot 7$ & $42 \cdot \overline{8}$ & 40.5 & 21.9 \\
\hline
\end{tabular}

*Human $11 \beta$-HSD1 is listed in the table for comparison.

decreased rapidly from day 6 , and were very weak after day 12 (Fig. 5). In adult tilapia, gonadal $11 \beta$-HSD2 showed a sexually dimorphic expression pattern. A single transcript of about $3.8 \mathrm{~kb}$ was detected in both sexes; however, the band was very intense in the testis but weaker in the ovary (Fig. 6). This is consistent with the tissue distribution revealed by RT-PCR. The $\sim 2.7$ and $\sim 3.8 \mathrm{~kb}$ transcript sizes detected in Northern blots were also consistent with the cloned eel and tilapia cDNA size, 2689 and 3804 bp respectively.

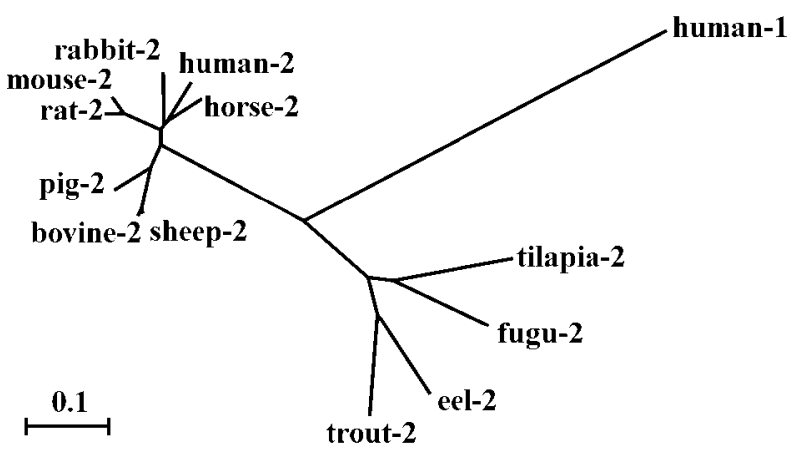

Figure 2 Phylogenetic tree of cloned fish and mammalian $11 \beta$-HSD2s. Human $11 \beta$-HSD1 was used as an outgroup. GenBank accession numbers are: horse (AF126744), human (S62789), rabbit (P51976), rat (NP_058777), mouse (CAA62219), sheep (AAA93156), cow (O77667), pig (AF374414), rainbow trout (BAC76709), fugu (http://www.ncbi.nlm.nih.gov/PMGifs/ Genomes/fugu.html), tilapia (AY190043) and Japanese eel (AB097668).

\section{Discussion}

Because of the low homology between the two types of mammalian $11 \beta$-HSD and the lack of information about which type may exist in fish testis, it is difficult to select two conserved regions present in both types of $11 \beta$-HSD that are suitable for PCR primers. Therefore, a method using oligonucleotides as screening probes was employed. By using mixed degenerate oligonucleotides corresponding to the sequence of a five amino acid region that is conserved in the two types of mammalian $11 \beta$-HSDs as an initial probe, a cDNA clone encoding $11 \beta$-HSD was isolated from Japanese eel testis cDNA library. Later, based on the eel $11 \beta$-HSD sequence, we designed degenerate primers to obtain an $11 \beta$-HSD fragment from Nile tilapia which was subsequently used as a probe to screen the cDNA library and obtain the full length tilapia $11 \beta$-HSD cDNA. Sequence analyses confirmed that this five amino acid region is conserved in 11 $\beta$-HSDs from fishes to mammals. Analysis of the predicted amino acid sequence indicated the proteins encoded by these two cDNAs are type 2-like $11 \beta$-HSDs, belonging to the short-chain alcohol dehydrogenase superfamily. Alignments of $11 \beta$-HSD2 amino acid sequences revealed two highly conserved motifs in both eel and tilapia $11 \beta$-HSD2 proteins which are characteristic of $11 \beta-H S D 2 s$. One is most likely the

\section{Female}

\section{Male}

\section{B P G H S L I O K M 2 B P G H S L I T K M $3+-$}

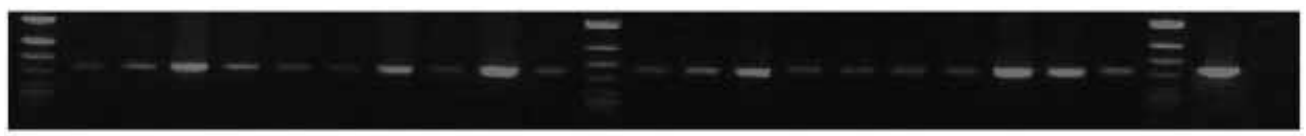

\section{- - - - - - -}

Figure 3 RT-PCR analysis of $11 \beta$-HSD2 from various tissues of adult tilapia. B, brain; $P$, pituitary; G, gill; $\mathrm{H}$, heart; S, spleen; L, liver; I, intestine; O, ovary; K, kidney; M, Muscle; T, testis; + and -, positive and negative controls; 1,2 and 3, markers. Lower panel, $\beta$-actin as internal control. 


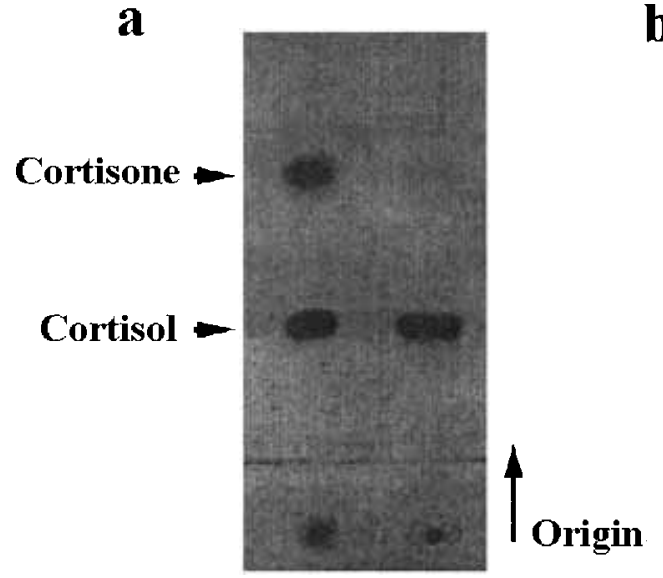

pBK-CMV/E11HSD pBK-CMV

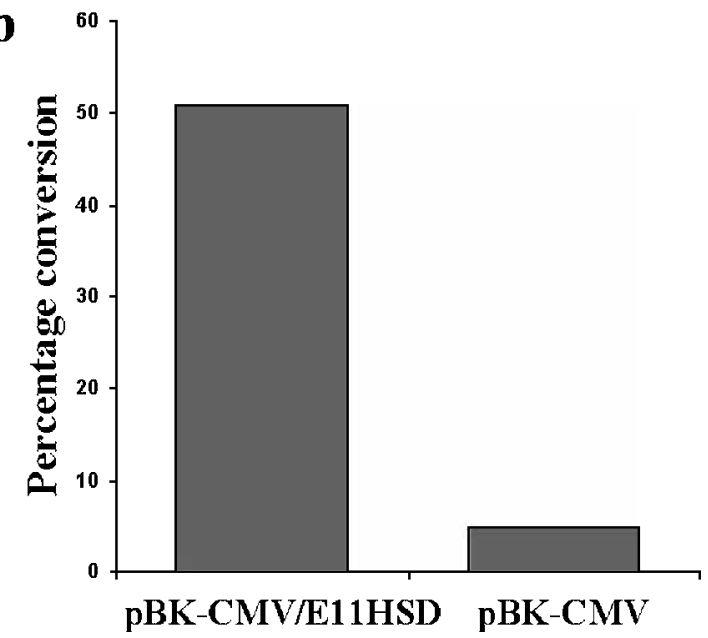

Figure 4 Enzyme assay validations for eel $11 \beta$-HSD. (a) Autoradiogram of thin-layer chromatography showing the conversion of cortisol to cortisone by COS-1 cells transfected by PBK-CMV/E11 HSD. Note COS-1 cells transfected by pBK-CMV (mock) did not catalyze the reaction. (b) Percentage conversion of ${ }^{3} \mathrm{H}$-labeled cortisol to cortisone by $\mathrm{COS}-1$ cells.
NAD-binding site. The other is probably important for catalytic activity. The amino acid sequences of Japanese eel and Nile tilapia 11ß-HSD2s show about $40-45 \%$ similarity to mammalian $11 \beta$-HSD2s, but only show $20-22 \%$ similarity to mammalian $11 \beta$-HSD1s. In the phylogenetic tree with human $11 \beta$-HSDl as an outgroup, both eel and tilapia $11 \beta$-HSDs were grouped into $11 \beta$-HSD2s.

A BLAST search with tilapia $11 \beta$-HSD2 nucleotide sequences in GenBank enabled us to isolate the fugu $11 \beta$-HSD2 genomic sequences. Fugu $11 \beta$-HSD2 protein sequences were obtained by locating the ORF and removing the intron sequences. The fugu $11 \beta-H S D 2$ gene consists of five exons interrupted by four introns, which is in accordance with the mammalian counterparts (Moore et al. 2000, Tomlinson \& Stewart 2001). All four introns are located in the same position as those of mammalian $11 \beta$-HSD2 (numbered and marked with arrows in Fig. 1), and have the typical intron characteristics, starting with GT ... and ending with ...AG. These data may indicate a conserved gene structure of $11 \beta$-HSD2 among vertebrates.

Notably, the eel $11 \beta$-HSD2 is 19 amino acids shorter than the other fish and mammalian $11 \beta$-HSD2s in the N-terminus (Fig. 1). It is unlikely that the cloned eel $11 \beta$-HSD2 is truncated because

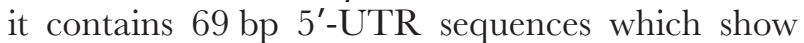

no homology to the N-terminus of other fish $11 \beta$-HSD2s. A region consisting of 47 amino acids in eel 11 $\beta$-HSD2 also cannot match those of other species including tilapia, fugu and rainbow trout. Thus, eel $11 \beta$-HSD2 is quite different in amino acid sequence compared with those of the other vertebrate $11 \beta$-HSD2s, but the significance of the changes to function, if any, remains unclear. Nevertheless, BLASTp of all fish $11 \beta$-HSD2 sequences including eel to GenBank resulted in high alignment (from 81 to $96 \%$ ) to the adh-short domain, the functional domain for the short-chain alcohol dehydrogenase superfamily. Unlike the fish $11 \beta$ HSD2s, none of the mammalian 11 $\beta$-HSD2 sequences showed higher than $77 \%$ similarities to the 252 residues of the adh-short domain. This is also in contrast to the mammalian $11 \beta-H S D 1 s$, which showed similarities higher than $80 \%$ to the 252 residues of the adh-short domain. Are these differences between fish and mammalian $11 \beta$ HSD2 responsible for functional differences between the two? Both display $11 \beta$-dehydrogenase activity. However, the fish $11 \beta$-HSD2 is also likely to be responsible for converting $11 \beta$ hydroxytestosterone to 11-KT.

Tilapia and eel 11 1 -HSD2 genes were found to be expressed in a wide variety of tissues, which is more similar to the tissue distribution of mammalian $11 \beta$-HSD1 than of $11 \beta$-HSD2. A similar pattern of expression of $11 \beta$-HSD2 was 

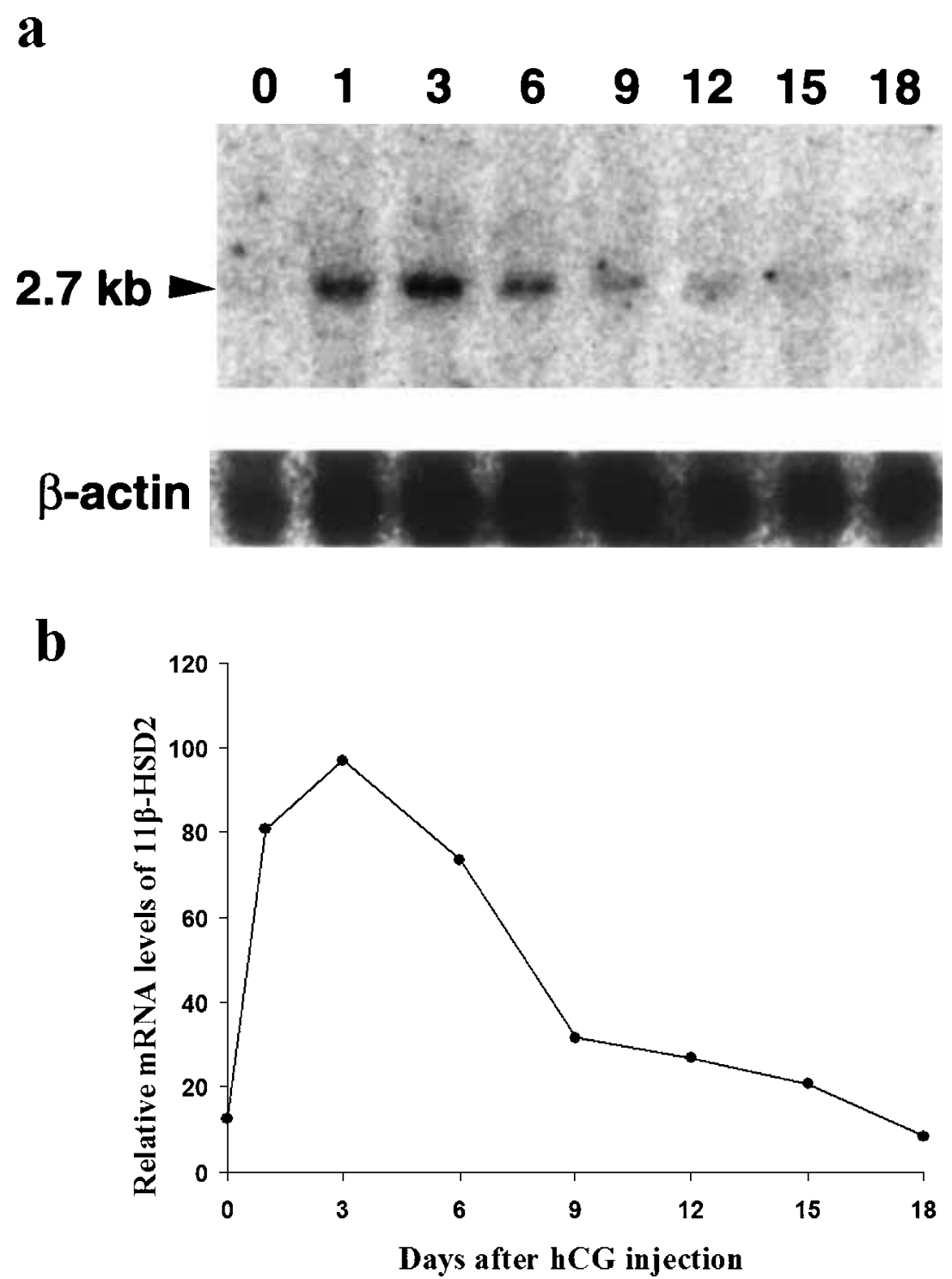

Figure 5 Northern blot analysis of the expression of $11 \beta-H S D 2$ in eel testis after $\mathrm{hCG}$ induction. (a) The ${ }^{32} \mathrm{P}$-labeled eel $11 \beta-H S D$ cDNA was hybridized with $10 \mu \mathrm{g}$ total RNA from 0 , untreated eels and 1-18, eels 1-18 days after hCG injection (upper panel). The lower panel shows the same membrane after stripping and hybridization with eel $\beta$-actin probe. (b) The expression levels were normalized to $\beta$-actin and expressed as the percentage of respective controls.

found in rainbow trout (Kusakabe et al. 2003). In mammals, aldosterone acts via mineralocorticoid receptors (MRs) to control salt and water flux in epithelial organs such as the kidney and colon to maintain circulatory homeostasis. Inappropriate glucocorticoid-mediated activation of MRs in aldosterone target tissues is prevented by the glucocorticoid metabolizing enzyme $11 \beta$-HSD2
(Moore et al. 2000). In tilapia, RT-PCR analysis of tissue distribution revealed a high level of expression of $11 \beta$-HSD2 in the kidney (including the cortisol synthesizing interrenal cells), gill and intestine, the most important organs for maintenance of circulatory homeostasis. This seems to suggest that fish $11 \beta$-HSD2 might function in the same way as the mammalian counterparts. 

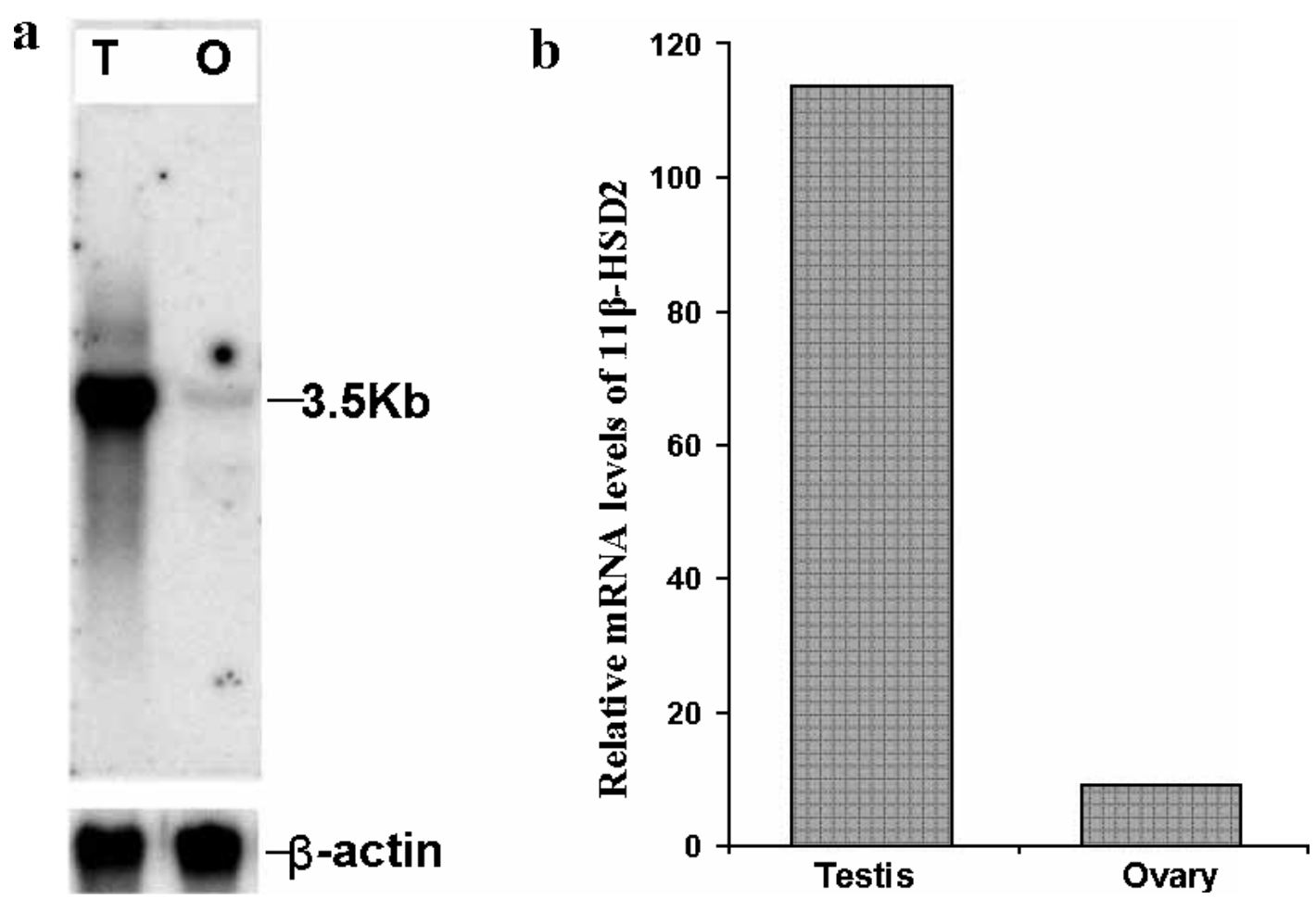

Figure 6 Northern blot analysis of the expression of $11 \beta-H S D 2$ in tilapia gonads. (a) The ${ }^{32} \mathrm{P}$-labeled tilapia $11 \beta$-HSD cDNA fragment was hybridized with $5 \mu \mathrm{g}$ mRNA from testis $(T)$ and ovary $(\mathrm{O})$. The lower panel shows the same membrane after stripping and hybridization with the tilapia $\beta$-actin probe. (b) The expression levels were normalized to $\beta$-actin and expressed as the percentage of respective controls.

However, although a mineralocorticoid receptor exists in rainbow trout (Colombe et al. 2000), its native ligand is unclear, since aldosterone is generally absent in teleosts.

Transient expression of pE11 HSD in COS-1 cells showed that the protein encoded by this cDNA has dehydrogenase activity typical of 11ß-HSD2. COS-1 cells transfected by the recombinant expression vector $\mathrm{pBK}-\mathrm{CMV} / \mathrm{E} 11$ HSD could efficiently convert cortisol to cortisone in only two hours of incubation (Fig. 4). In addition, this eel $11 \beta$-HSD2 could also convert corticosterone to 11-dehydrocorticosterone when expressed in COS-1 cells (data not shown). In mammals, two isoforms of the enzyme $11 \beta$ hydroxysteroid dehydrogenase interconvert the active glucocorticoid, cortisol, and inactive cortisone. Whether fish has both types or has only one

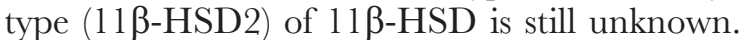

In tilapia, the highest expression level of $11 \beta$-HSD2 was found in the testis. On the contrary, the expression level in the ovary was very low (Fig. 3). This sex dimorphic expression pattern was also confirmed by Northern blotting (Fig. 6). Therefore, it seems likely that fish $11 \beta$-HSD2 also functions to convert $11 \beta$-hydroxytestosterone to $11-\mathrm{KT}$, the main androgen found in the majority of fish species. The expression of the $11 \beta$-HSD gene in eel testis detected by Northern blotting appeared to be induced by hGG injection. In untreated eels, no obvious $11 \beta$-HSD2 transcripts were detected. This is different from results on tilapia, in which $11 \beta$-HSD2 transcripts were dominantly found in testis. This difference might be explained by the fact that tilapia mature naturally under laboratory and aquaculture conditions whereas eels cannot start spermatogenesis and reach maturation under these conditions without hormone treatment. One day after hCG injection, strong hybridization signals were detected from eel testicular RNA. The signal intensity peaked in samples from eels three days after hCG treatment. From day 6, the hybridization signal started to decrease. The time course of such expression was consistent with that 
of hCG-induced changes in serum 11-KT levels of eels (Miura et al. 1991a), indicating the importance of $11 \beta$-HSD activity in hGG-induced 11-KT synthesis in testis. From results of this study and the previous study on $\mathrm{P} 450$ (11 $\beta$ ) (Jiang et al. 1998), it is obvious that the hCG-induced $11-\mathrm{KT}$ production in Japanese eels involves enhanced testicular expression of both the $\mathrm{P} 450(11 \beta)$ gene and the $11 \beta$-HSD gene. Increased transcripts from both genes may contribute to an increase in enzyme proteins and activities, which lead to increased 11-KT synthesis in hCG-treated Japanese eels. Very recently, the ability of fish $11 \beta-\mathrm{HSD} 2$ to convert $11 \beta$-hydroxytestosterone to $11-\mathrm{KT}$ was demonstrated using recombinant rainbow trout 11ß-HSD2 (Kusakabe et al. 2003). Unlike tilapia, both ovary and testis of rainbow trout show relatively high expression of $11 \beta$-HSD2. Transcripts were localized to testis Leydig cells and theca and granulosa cells of the ovarian follicle. A strong seasonal pattern of expression of $11 \beta$-HSD2 was found in rainbow trout gonads, but unlike this study, no strong relationship was found between circulating 11-KT levels and testis 11ß-HSD2 mRNA levels.

In summary, $11 \beta$-HSD2 cDNAs were cloned from the testes of the Japanese eel and Nile tilapia. These $11 \beta$-HSD 2 genes were expressed in a wider range of tissues than mammalian $11 \beta$-HSD2s. Similar to mammals, fish $11 \beta$-HSD2 appeared to act exclusively as an NAD-dependent dehydrogenase, inactivating cortisol (or corticosterone) to cortisone (or 11-dehydrocorticosterone). Most importantly, like rainbow trout $11 \beta$-HSD2, eel and tilapia $11 \beta$-HSD2s may also have the ability to convert $11 \beta$-hydroxytestosterone to $11-\mathrm{KT}$, the main androgen found in the majority of fish species, and therefore expression of the $11 \beta$-HSD2 gene in testes may play an important role in spermatogenesis.

\section{Acknowledgements}

This work was supported, in part, by Grants-inAids for Research from CREST, JST (Japan Science Technology Corporation) and Bio Design Program from the Ministry of Agriculture, Forestry and Fisheries, Japan. D S W is grateful to Japan Society of Promotion of Science (JSPS) for a fellowship.

\section{References}

Agarwal AK, Monder C, Eckstein B \& White PC 1989 Cloning and expression of rat cDNA encoding corticosteroid $11 \beta$-dehydrogenase. Fournal of Biological Chemistry 264 18939-18943.

Agarwal AK, Mune T, Monder C \& White PC $1994 \mathrm{NAD}^{+}$dependent isoform of $11 \beta$-hydroxysteroid dehydrogenase. Fournal of Biological Chemistry 269 25959-25962.

Albiston AL, Obeyesekere VR, Smith RE \& Krozowski ZS 1994 Cloning and tissue distribution of the human $11 \beta$-hydroxysteroid dehydrogenase type 2 enzyme. Molecular and Cellular Endocrinology 105 R $11-R 17$

Cole TJ 1995 Cloning of the mouse $11 \beta$-hydroxysteroid dehydrogenase type 2 gene: tissue specific expression and localization in distal convoluted tubules and collecting ducts of the kidney. Endocrinology 136 4693-4696.

Colombe L, Fostier A, Bury N, Pakdel F \& Guiguen Y 2000 A mineralocorticoid-like receptor in the rainbow trout, Oncorhynchus mykiss: cloning and characterization of its steroid binding domain. Steroids 65 319-328.

Jiang JQ, Kobayashi T, Ge W, Kobayashi H, Tanaka M \& Nagahama Y 1996 Fish testicular 11 $\beta$-hydroxylase: cDNA cloning and expression during spermatogenesis. FEBS Letters 397 250-252.

Jiang JQ, Young G, Kobayashi T \& Nagahama Y 1998 Eel (Anguilla japonica) testis $\beta$-hydroxylase gene is expressed in interrenal tissue and its product lacks aldosterone synthesizing activity. Molecular and Cellular Endocrinology 146 207-211.

Krozowski ZS, Provencher PH, Smith RE, Obeyesekere VR, Mercer WR \& Albiston AL 1994 Isozymes of 11ß-hydroxysteroid dehydrogenase: which enzyme endows mineralocorticoid specificity? Steroids 59 116-120.

Kusakabe M, Kobayashi T, Todo T, Mark Lokman P, Nagahama Y \& Young G 2002 Molecular cloning and expression during spermatogenesis of a cDNA encoding testicular $11 \beta$-hydroxylase $(\mathrm{P} 45011 \beta)$ in rainbow trout (Oncorhynchus mykiss). Molecular Reproduction and Development 62 456-469.

Kusakabe M, Nakamura I \& Young G $200311 \beta$-Hydroxysteroid dehydrogenase complementary deoxyribonucleic acid in rainbow trout: cloning, sites of expression, and seasonal changes in gonads. Endocrinology $1442534-2545$.

Liu S, Govoroun M, D'Cotta H, Ricordel MJ, Lareyre JJ, McMeel OM, Smith T, Nagahama Y \& Guiguen Y 2000 Expression of cytochrome $\mathrm{P} 45011 \beta$ (11ß-hydroxylase) gene during gonadal sex differentiation and spermatogenesis in rainbow trout, Oncorhynchus mykiss. Fournal of Steroid Biochemistry and Molecular Biology 75 291-298.

Miura T, Yamauchi K, Takahashi H \& Nagaham Y $1991 a$ Induction of spermatogenesis in male Japanese eel, Anguilla japonica, by a single injection of human chorionic gonadotropin. Zoological Science 8 63-73.

Miura T, Yamauchi K, Takahashi H \& Nagahama Y 1991b Human chorionic gonadotropin induces all stages of spermatogenesis in vitro in the male Japanese eel (Anguilla japonica). Developmental Biology 146 258-262.

Miura T, Yamauchi K, Takahashi H \& Nagahama Y 1991c Hormonal induction in vitro of all stages of spermatogenesis in the male Japanese eel (Anguilla japonica). PNAS 88 5774-5778.

Monder C \& Lakshmi V 1989 Evidence of kinetically distinct forms of corticosteroid $11 \beta$-dehydrogenase in rat liver microsomes. fournal of Steroid Biochemistry 32 77-83.

Moore CC, Mellon SH, Murai J, Siiteri PK \& Miller WL 1993 Structure and function of the hepatic form of $11 \beta$-hydroxysteroid dehydrogenase in the squirrel monkey, an animal model of glucocorticoid resistance. Endocrinology 133 368-375.

Moore XL, Hoong I \& Cole TJ 2000 Expression of the $11 \beta$-hydroxysteroid dehydrogenase 2 gene in the mouse. Kidney International 57 1307-1312. 
Nagahama Y 1994 Endocrine regulation of gametogenesis in fish. International Fournal of Developmental Biology 38 217-229.

Naray-Fejes-Toth A \& Fejes-Toth G 1995 Expression cloning of the aldosterone target cell-specific $11 \beta$-hydroxysteroid dehydrogenase from rabbit collecting duct cells. Endocrinology 6 2579-2586.

Naray-Fejes-Toth A, Watlington CO \& Fejes-Toth G 1991 $11 \beta$-Hydroxysteroid dehydrogenase activity in the renal target cells of aldosterone. Endocrinology 129 17-21.

Rajan V, Chapman KE, Lyons V, Jamieson P, Mullins JJ, Edwards CR \& Seckl R 1995 Cloning, sequencing and tissue-distribution of mouse $11 \beta$-hydroxysteroid dehydrogenase-1 cDNA. Fournal of Steroid Biochemistry and Molecular Biology 52 141-147.

Romero DG, Zhou M \& Gomez-Sanchez CE 2000 Cloning and expression of the bovine $11 \beta$-hydroxysteroid dehydrogenase type-2. Foumal of Steroid Biochemistry and Molecular Biology 72 231-237.

Rusvai E \& Naray-Fejes-Toth A 1993 A new isoform of $11 \beta$-hydroxysteroid dehydrogenase in aldosterone target cells. Fournal of Biological Chemistry 268 10717-10720.

Tannin GM, Agarwal AK, Monder C, New MI \& White PC 1991 The human gene for $11 \beta$-hydroxysteroid dehydrogenase. Fournal of Biological Chemistry 266 16653-16658.
Thompson JD, Gibson TJ, Plewniak F, Jeanmougin F \& Higgins DG 1997 The ClustalX windows interface: flexible strategies for multiple sequence alignment aided by quality analysis tools. Nucleic Acids Research 24 4876-4882.

Tomlinson JW \& Stewart PM 2001 Cortisol metabolism and the role of $11 \beta$-hydroxysteroid dehydrogenase. Best Practice and Research Clinical Endocrinology and Metabolism 15 61-78.

Yang K, Smith CL, Dales D, Hammond GL \& Challis JR 1992 Cloning of an ovine 11ß-hydroxysteroid dehydrogenase complementary deoxyribonucleic acid: tissue and temporal distribution of its messenger ribonucleic acid during fetal and neonatal development. Endocrinology 131 2120-2126.

Zhou MY, Gomez-Sanchez EP, Cox DI, Cosby D \& Gomez-Sanchez GE 1995 Cloning, expression, and tissue distribution of the rat nicotinamide adenine dinucleotidedependent $11 \beta$-hydroxysteroid dehydrogenase. Endocrinology 136 3729-3734.

Received in final form 1 July 2003

Accepted 11 July 2003

Made available as an Accepted Preprint 11 July 2003 